\title{
Modeling Rectangular Cantilevers during Torsion and Deflection for Application to Frictional Force Microscopy
}

\author{
Victor C. Hayden and Luc Y. Beaulieu* \\ Department of Physics and Physical Oceanography, Memorial University of Newfoundland, St. John's Newfoundland, Canada
}

\begin{abstract}
A numerical and experimental analysis of the optical beam deflection system used to monitor microcantilevers subjected to simultaneous deflection and twisting such as in lateral or frictional force microscopy was performed. This study focused on two optical beam deflection orientations where in the first case the optical beam and the detector are at a right angle to the length of the cantilever and the second case, which is the more standard orientation, the optical beam is parallel to the length of the lever. This study finds that it is possible to model the twist and the deflection separately and treat each motion independently. Simulations have shown that the above-mentioned systems are equivalent in accuracy and sensitivity for monitoring the simultaneous twist and deflection of cantilevers.
\end{abstract}

Key words: frictional force microscopy, lateral force microscopy, atomic force microscopy, microcantilevers, numerical modeling

\section{INTRODUCTION}

The atomic force microscope (Binnig et al., 1986) is used for a wide variety of tasks such as surface imaging, mapping out different electrical and magnetic regions, differentiation between areas of different friction, etc. In this last application, often referred to as lateral or frictional force microscopy (FFM), the twisting of the cantilever is monitored to measure the drag force between the sample and the tip (Mate et al., 1987). There have been many applications of FFM in the last two decades from studying surface coatings, investigating the effects of lubricants to understanding basic friction, etc. (Ruan \& Bhushan, 1994; Mate, 1995; Goto et al., 2003).

Of primary importance in obtaining quantitative FFM measurements is to properly calibrate the instrument and to obtain an accurate value of the normal and lateral spring constants of the lever (Ogletree et al., 1996; Lantz et al., 1997; Varenberg et al., 2003; Tocha et al., 2006). In most situations the basic setup of the FFM has followed the original design of the first atomic force microscopes (AFMs), which were capable of performing both surface imaging and frictional analysis (Meyer \& Amer, 1988, 1990). In such a case the optical beam deflection system and the major axis of the cantilever all lie on the same geometric plane in space. This corresponds to the case where $\phi=180^{\circ}$ in Figure 1. Recently an alternate geometry has been used in metrology and cantilever sensor instruments to monitor

Received October 9, 2008; accepted March 19, 2009

*Corresponding author. E-mail: lbeaulieu@mun.ca the deflection and/or twist of the cantilevers (Godin et al., 2003; Yacoot et al., 2007). In this alternate geometry the optical beam deflection system is at a right angle with respect to the cantilever. This corresponds to the case where $\phi=90^{\circ}$ in Figure 1.

In this article we investigate the difference in sensitivity of the optical beam deflection system; $\phi=90^{\circ}$ and $\phi=180^{\circ}$ for cantilevers subjected to torsional and vertical displacements. In a method similar to that reported by us in a previous work (Beaulieu et al., 2006, 2007), we have modeled the optical beam deflection system based on simple geometrical optics. The model was then tested using a macroscopic cantilever that allows the variables of the system to be measured with a higher accuracy compared to using actual microcantilevers such as those used for AFM and FFM.

\section{Materials and Methods}

\section{Numerical Model}

The model is based on the schematic diagram of the cantilever/optical beam deflection system shown in Figure 1. In this diagram, the cantilever surface is in the $x-y$ plane and is orientated in the positive $x$ direction. An incident laser hits the undeflected cantilever at the point $\left(D_{x}, D_{y}, 0\right)$ measured from the base of the cantilever chip. The incident laser is fixed at an angle of inclination $\theta$ with respect to the $x-y$ plane and at an azimuthal angle $\phi$ measured from the positive $x$-axis. The laser reflects from 


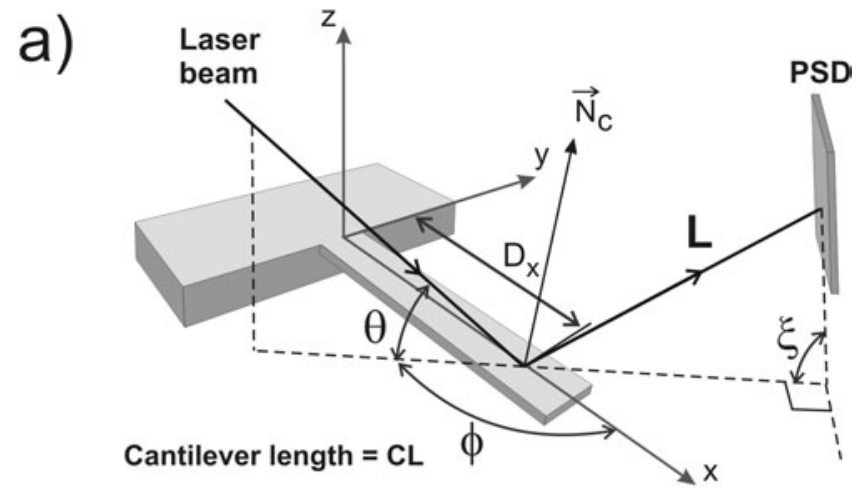

b)

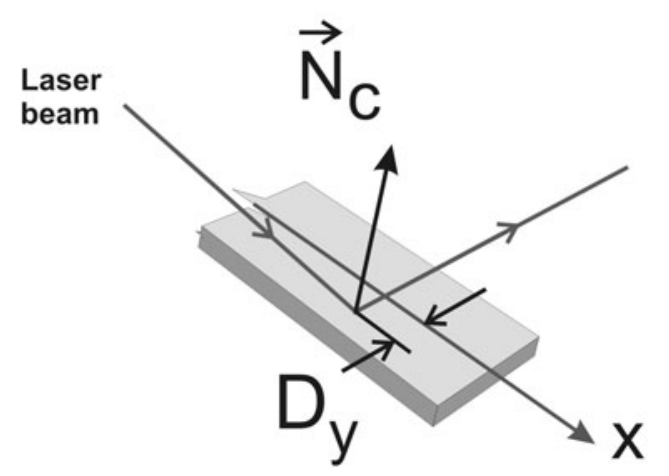

Figure 1. a: Schematic representation of the cantilever/optical beam deflection system. The incident laser inclined at an angle $\theta$ with an azimuthal angle $\phi$ is made incident on the undeflected cantilever at the point $\left(D_{x}, D_{y}, 0\right)$. The reflected laser then impinges on a PSD detector a distance $L$ from the lever. The vector $\mathbf{N}_{\mathbf{c}}$ is the surface normal of the cantilever at the laser/cantilever intersection. b: Close up view of the free end of the cantilever showing the offset $D_{y}$.

the free end of the cantilever onto a position sensitive detector (PSD) held at an initial distance $L$ from the cantilever. The length of the cantilever is given by $C L$. The PSD is itself inclined at an angle $\xi$ also with respect to the $x-y$ plane. In Figure 1, the line labeled $\mathbf{N}_{\mathrm{c}}$ is the vector normal to the surface of the cantilever and is used to calculate the direction of the reflected laser beam in accordance with the law of reflection.

The numerical model used in this work is similar to the model developed by us in a previous work (Beaulieu et al., 2006, 2007). In short, the optical beam was described by a vector line of the form

$$
\mathbf{I}=\mathbf{I}_{\mathbf{p}}+t \mathbf{I}_{a}
$$

where $\mathbf{I}_{\mathbf{p}}$ is any point on the line, $\mathbf{I}_{\mathbf{a}}$ is the unit direction vector, and $t$ is any scalar. In this case $\mathbf{I}_{\mathbf{p}}$ is defined as the initial point where the incident laser hits the cantilever at $\mathbf{I}_{\mathbf{p}}=\left(D_{x}, D_{y}, 0\right)$. Given the geometry shown in Figure 1, the direction vector $\mathbf{I}_{\mathbf{a}}$ is defined as

$$
\mathbf{I}_{\mathbf{a}}=(\cos (\theta) \cos (\pi-\phi), \cos (\theta) \sin (\pi-\phi),-\sin (\theta)),
$$

where in this work only the azimuthal angles $\phi=90^{\circ}$ and $\phi=180^{\circ}$ are considered. To determine the direction of the reflected beam, the surface normal $\mathbf{N}_{\mathbf{c}}$ of the cantilever at the cantilever/optical beam intersection must be calculated.

Here, the cantilever was modeled by describing the vertical deflection and lateral twist independently. It is assumed that the deflection of the cantilever does not affect the angular orientation of the lever. During atomic force microscopy, the cantilever is deflected by a point load applied near the end of the lever. In such a case the curvature of the cantilever is described by (Sarid, 1994)

$$
z(x)=\frac{F x^{2}}{6 E I}(x-3 a)
$$

where $F$ is the applied point force, $E$ is the Young's modulus of elasticity, $I$ is the area moment of inertia, and $a$ is the position of the applied force. To determine the torsional component of the normal vector, the twist of the cantilever must be defined. The angle of the cantilever $\theta_{C L}$, subjected to a torque $T$, at any point $x$ along the lever is given by (Reissner \& Stein, 1951)

$\begin{aligned} \theta_{C L}=\frac{T \cdot C L}{2(1-\mu) D w} & {\left[\frac{x}{C L}-\frac{\sinh \left(\frac{4 \lambda x}{C L}\right)}{4 \lambda}\right.} \\ & \left.-\frac{\tanh (4 \lambda)}{4 \lambda}\left(1-\cosh \left(\frac{4 \lambda x}{C L}\right)\right)\right],\end{aligned}$

where $C L$ is the length of the cantilever, $w$ is the width of the cantilever, and $\mu$ is Poisson's ratio. $D$ is the local flexural stiffness given by

$$
D=\frac{E h^{3}}{12\left(1-\mu^{2}\right)},
$$

where $E$, as before, is the Young's modulus of elasticity of material of the cantilever of thickness $h$ and $\lambda$ is the aspect-ratio parameter given by

$$
\lambda=\frac{C L}{w} \sqrt{\frac{3}{2}(1-\mu)} .
$$

In this work the twist of the cantilever at the free end is controlled. Solving equation (4) for the twist at the tip $(x=C L)$ yields 


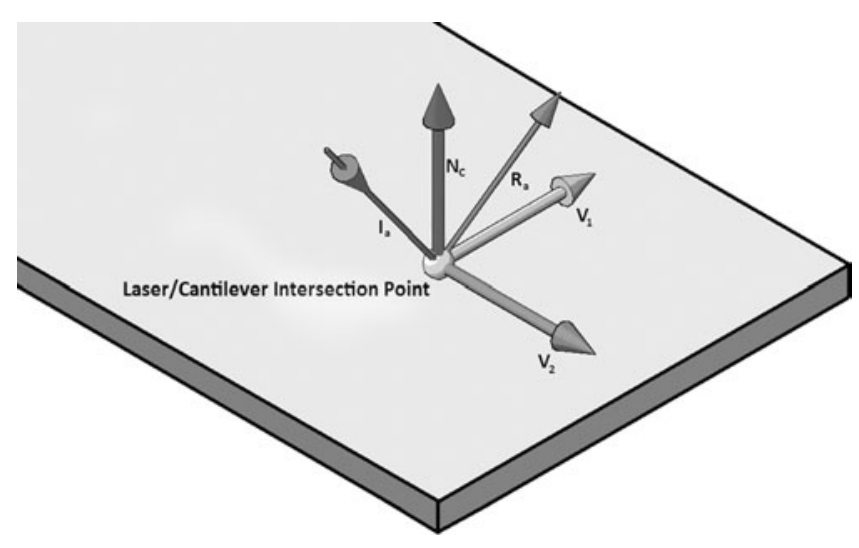

Figure 2. Schematic representation of the free end of the cantilever indicating the positions of the incident laser vector $\mathbf{I}_{\mathbf{a}}$, the reflected laser $\mathbf{R}_{\mathbf{a}}$, the surface normal $\mathbf{N}_{\mathbf{c}}$, and the tangential and longitudinal surface vectors $\mathbf{V}_{1}$ and $\mathbf{V}_{2}$.

$$
\theta_{C L}(C L)=\frac{T \cdot C L}{2(1-\mu) D w}\left[1-\frac{\tanh (4 \lambda)}{4 \lambda}\right]
$$

where the only unknown in this equation is $T$. Solving equation (7) for $T$ gives

$$
T=\frac{\theta_{C L}(C L)}{C L}\left(\frac{8 \lambda(1-\mu) D w}{4 \lambda-\tanh (4 \lambda)}\right)
$$

Hence, to calculate the local twist angle at any position along the cantilever from the twist angle at the free end, it is necessary to use equations (4), (5), (6), and (8).

Given an angular and lateral deflection obtained by equations (3) and (4), the normal vector $\mathbf{N}_{\mathbf{c}}$ at the cantilever/ incident laser intersection can be determined. The normal vector $\mathbf{N}_{\mathbf{c}}$ (shown schematically in Fig. 2) was found by taking the derivatives of equations (3) and (4) and evaluating them at the cantilever/incident laser intersection point. As shown in Figure 2, $\mathbf{V}_{\mathbf{l}}$ is the vector indicating the tangent to the cantilever surface in a direction perpendicular to the lever's length. $\mathbf{V}_{\mathbf{1}}$ is given by

$$
\begin{aligned}
& V_{1 x}=0 \\
& V_{1 y}=1 \\
& V_{1 z}=-\tan \left(\theta_{C L}\right) .
\end{aligned}
$$

The vector $\mathbf{V}_{2}$ shown in Figure 2 is obtained from the first derivative of equation (3):

$$
\begin{aligned}
& V_{2 x}=1 \\
& V_{2 y}=0 \\
& V_{2 z}=\frac{\partial z}{\partial x}=2 \gamma x_{o}\left(x_{o}-3 a\right)+\gamma x_{o}^{2} .
\end{aligned}
$$

a)

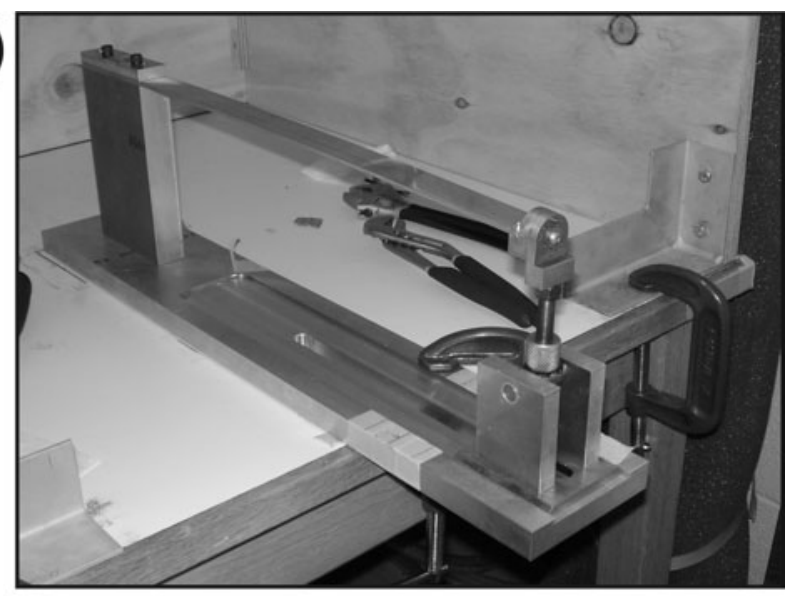

b)

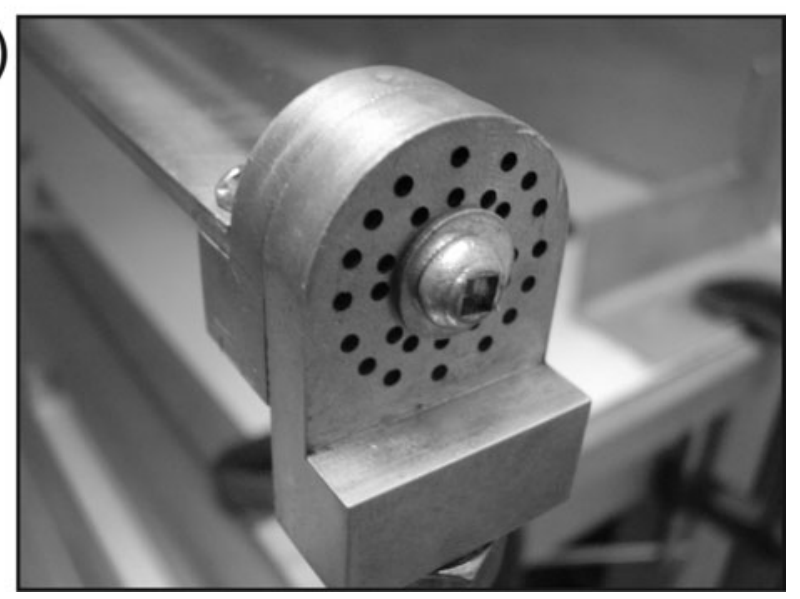

Figure 3. a: Photograph of the macroscopic cantilever used in this work. b: Photograph of the indexing head used to torque the free end of the cantilever.

The vectors $\mathbf{V}_{\mathbf{1}}$ and $\mathbf{V}_{\mathbf{2}}$ are used to define the normal vector by

$$
\vec{N}_{c}=\frac{\vec{V}_{1}}{\left\|\vec{V}_{1}\right\|} \times \frac{\vec{V}_{2}}{\left\|\vec{V}_{2}\right\|}
$$

where $\left\|\vec{V}_{i}\right\|$ is the length of the vector $\vec{V}_{i}$. Once the normal vector $\mathbf{N}_{\mathbf{c}}$ is determined, the rest of the information necessary to find the reflected laser/detector intersection is as outlined in detail in the following (Beaulieu et al., 2006, 2007).

\section{Equipment}

To test the results of our model, a macrocantilever with an indexing head located at the free end, as shown in Figure 3, was designed and built. The lever was made from a $410 \times$ $25.4 \times 3.14 \mathrm{~mm}$ piece of aluminum held rigidly at one end and attached to an indexing head at the other end, which also served to deflect the cantilever in the positive or nega- 
a)
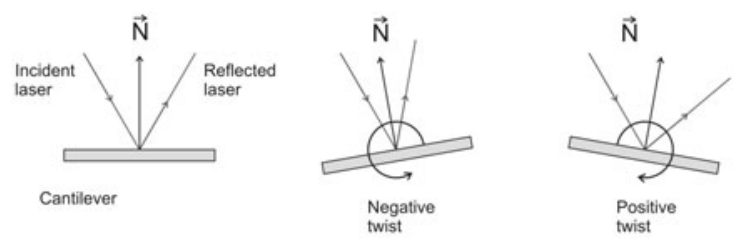

b)

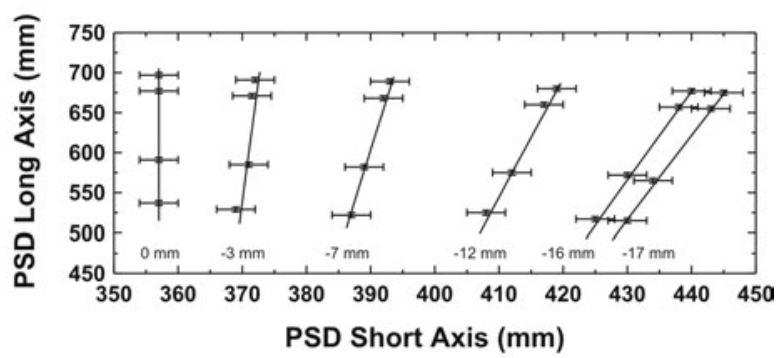

c)

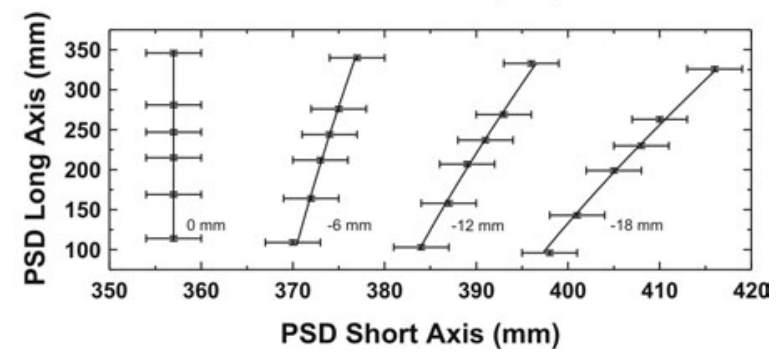

Figure 4. a: Schematic representation showing the relationship between the incident and reflected laser when the cantilever is subjected to a negative and positive twist. b: Experimental data (points) and calculated fits (solid curves) for a cantilever subjected to a negative twist from 0 to $13.5^{\circ}$ and deflected by $0,-3,-7$, $-12,-16$, and $-17 \mathrm{~mm}$. b: Experimental data (points) and calculated fits (solid curves) for a cantilever subjected to a positive twist from 0 to $13.5^{\circ}$ and deflected by $0,-6,-12$, and $-18 \mathrm{~mm}$.

tive $z$-direction. The indexing head was machined from two pieces of brass and was designed to apply a torque to the cantilever by rotating the two pieces until holes made to accept a dowel pin lined up. The angle of the cantilever was also verified by a digital angle gauge (King Canada, KW180). To set the deflection of the cantilever, a threaded rod was inserted below the indexing head in conjunction with a pair of locking nuts used to fix the cantilever in place. This system allowed the cantilever to be deflected and twisted simultaneously.

A standard presentation type laser pointer was used to simulate the incident laser on the cantilever. The laser was reflected from the free end of the cantilever, which was polished to a mirror finish, onto a graph paper placed on a board of wood used to simulate a PSD.

\section{Results}

Figure $4 \mathrm{~b}, \mathrm{c}$ shows experimental data points along with calculated curves (solid lines) obtained from our model for

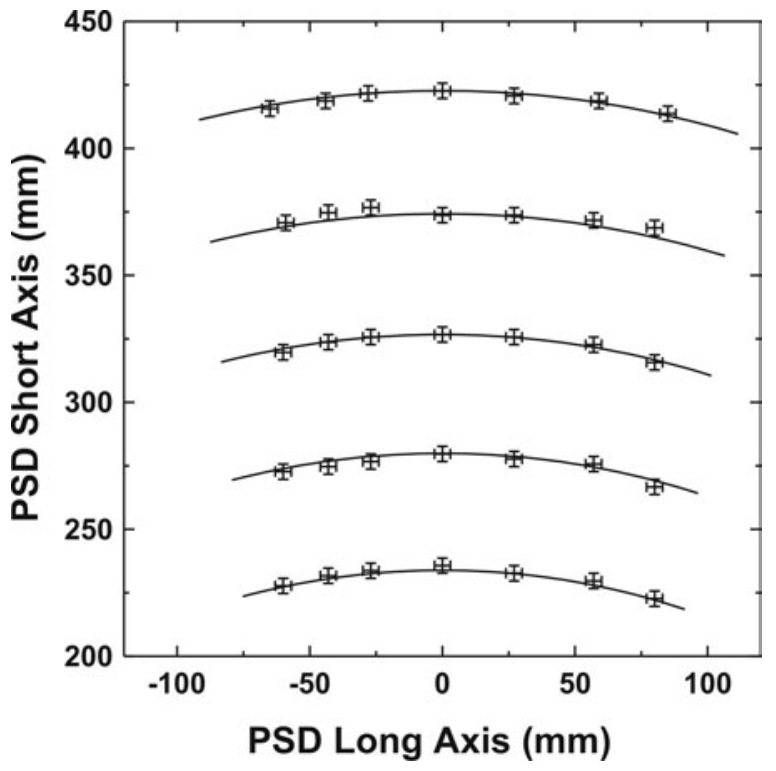

Figure 5. Experimental data (points) and calculated fits (solid curves) for a cantilever subjected to a negative twist from 0 to $\pm 15^{\circ}$ and deflected by $0, \pm 6$, and $\pm 12 \mathrm{~mm}$.

the following configuration: $\theta=57.5^{\circ}, \xi=70^{\circ}, \phi=90^{\circ}$, $D_{x}=357 \mathrm{~mm}, D_{y},=-1 \mathrm{~mm}$, and $L=569 \mathrm{~mm}$. The data shown in Figure $4 \mathrm{~b}$ were taken while the cantilever was subjected to a twist in the opposite direction as the optical beam referred to here as a negative twist, as illustrated in Figure 4a. As can be seen, the experimental results match the predicted curves obtained from the model. The macrocantilever was twisted from 0 to $13.5^{\circ}$ with vertical deflections of $0,-3,-7,-12,-16$, and $-17 \mathrm{~mm}$, where the negative sign implies a downward cantilever deflection. The error bars on the experimental data points represent the size of the laser spot on the board simulating the PSD. The data shown in Figure 4c show experimental data points along with calculated curves (solid lines) obtained from our model while the cantilever was subjected to a positive twist. The macrocantilever was twisted from 0 to $13.5^{\circ}$ with vertical deflections of $0,-6,-12$, and $-18 \mathrm{~mm}$. As in the previous case, the calculated curves fit the experimental results very well. It should be made clear that the model has no adjustable parameters and is uniquely determined by the variables of the system $\theta, \xi, \phi, D_{x}, D_{y}, C L$, and $L$.

Figure 5 shows experimental data points along with calculated curves (solid lines) obtained from our model for the following configuration: $\theta=22^{\circ}, \xi=90^{\circ}, \phi=90^{\circ}, D_{x}=$ $340 \mathrm{~mm}, D_{y}$, $=0 \mathrm{~mm}$, and $L=872 \mathrm{~mm}$. Similar to the $\phi=$ $90^{\circ}$ system, the results from the model match very well the experimental results obtained with the macrocantilever. From Figures 4 and 5 it is clear that the model developed properly characterizes the simultaneous deflection and twist of a rectangular cantilever. With this model it is now possible to make predictions as to which system is better suited for use in FFM. 


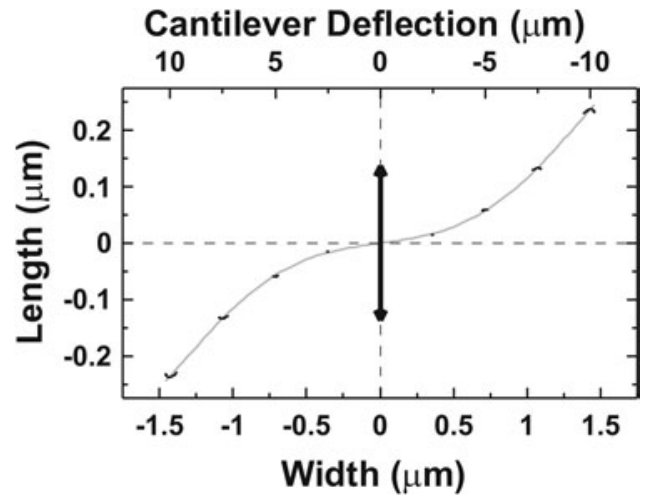

Figure 6. Calculated curves showing the motion of laser spot on the cantilever for the $\theta=80^{\circ}, \xi=10^{\circ}, \phi=90^{\circ}$ (light and dark curves) and $\phi=180^{\circ}$ (bold arrow), $C L=400 \mu \mathrm{m}, D_{x}=350 \mu \mathrm{m}$, and $D_{y}=0, L=2 \mathrm{~cm}$ configuration. The light solid curve shows the motion of the laser spot on the cantilever while the latter is being deflected from -10 to $10 \mu \mathrm{m}$. The smaller darker curves show the motion of the laser spot on the cantilever while the cantilever is deflected to a fixed point and twisted $\pm 10^{\circ}$. The dark arrow shows the motion of the laser spot in the $\phi=180^{\circ}$ system during deflection and twist.

It is also possible to monitor the motion of the laser spot on the cantilever during cantilever twisting and deflection. Figure 6 shows a plot of how the incident optical beam travels along the cantilever while the latter undergoes deflections from -10 to $+10 \mu \mathrm{m}$ for a system in the following configuration: $\theta=80^{\circ}, \xi=10^{\circ}, \phi=90^{\circ}$ (light and dark curves) and $\phi=180^{\circ}$ (solid arrow), $C L=400 \mu \mathrm{m}, D_{x}=350$ $\mu \mathrm{m}$, and $D_{y}=0, L=2 \mathrm{~cm}$ with zero twist. This configuration is consistent with actual sizes used in various commercial AFM instruments. The ordinate in Figure 6 represents a section along the length of the free end of the cantilever. The bottom abscissa represents the width of the cantilever while the top abscissa indicates the deflection of the lever. For $D_{y}=0 \mu \mathrm{m}$ the path of the incident laser for the $\phi=$ $180^{\circ}$ configuration will always remain along a straight line in the center of the lever. However, the path of the $\phi=90^{\circ}$ configuration follows a more complicated path when the cantilever is twisted and deflected. More specifically, the light curve in Figure 6 shows the motion of the cantilever in the $\phi=90^{\circ}$ configuration while the lever is deflected by $\pm 10 \mu \mathrm{m}$ with zero twist. The smaller darker curves show the motion of the laser spot on the cantilever while the cantilever is deflected by the amount indicated by the top abscissa and twisted by $\pm 10^{\circ}$. From these data it is clear that despite extreme twisting and deflections the center of the incident laser remains on the cantilever surface for both the $\phi=90^{\circ}$ and $\phi=180^{\circ}$ configuration.

The orientation of the system influences the size and shape of the required PSD. The trace of the reflected laser on the PSD for the configurations $\theta=80^{\circ}, \xi=10^{\circ}, C L=$ $400 \mu \mathrm{m}, D_{x}=350 \mu \mathrm{m}, D_{y}=0 \mu \mathrm{m}$, and $L=2 \mathrm{~cm}$ is shown a)

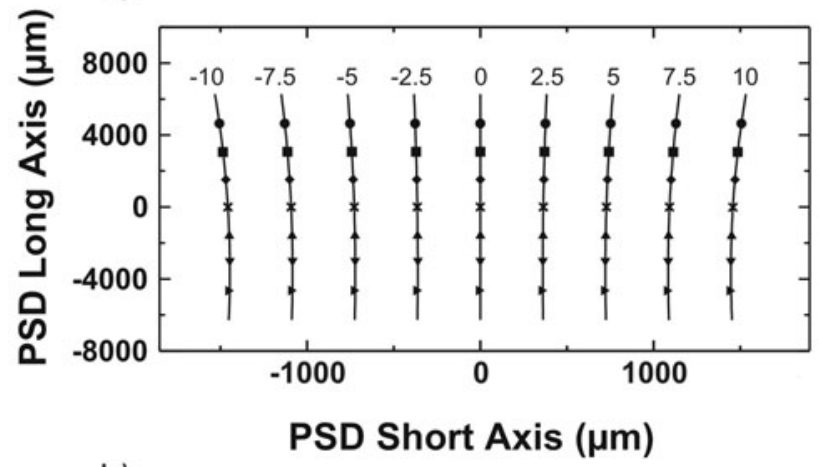

b)

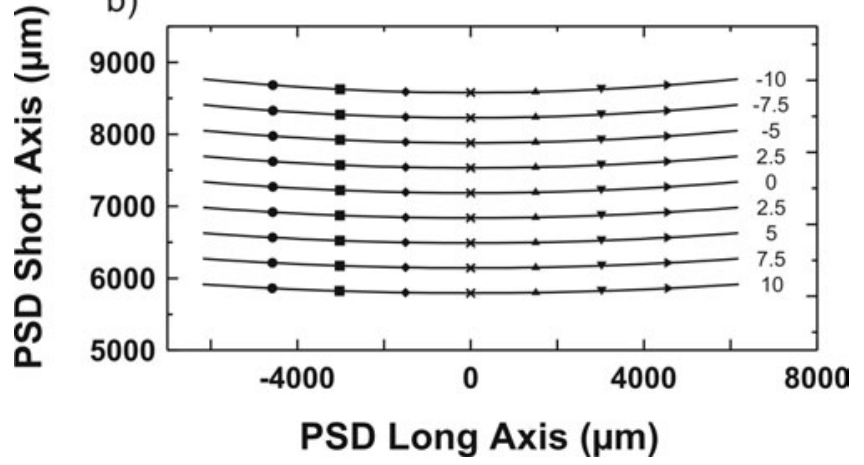

Figure 7. Experimental data (points) and calculated fits (solid curves) for a cantilever twisted from 0 to $\pm 10^{\circ}$ and deflected by 0 , $\pm 2.5, \pm 5, \pm 7.5$, and $\pm 10 \mathrm{~mm}$ with (a) $\phi=90^{\circ}$ and (b) $\phi=180^{\circ}$. In this figure the symbols represent the following twist: $0=$ $-7.5^{\circ}, \boldsymbol{\square}=-5^{\circ}, \nabla=-2.5^{\circ}, \boldsymbol{\varkappa}=0^{\circ}, \boldsymbol{\Delta}=2.5^{\circ}, \boldsymbol{\nabla}=5^{\circ}, \boldsymbol{\nabla}=7.5^{\circ}$.

in Figures $7 \mathrm{a}$ and $7 \mathrm{~b}$ for $\phi=90^{\circ}$ and $\phi=180^{\circ}$, respectively. In Figure 7 the cantilever was deflected at a fixed position from -10 to $10 \mu \mathrm{m}$ and twisted from $-10^{\circ}$ to $10^{\circ}$. From Figure 7a, it can be seen that the PSD for the $\phi=90^{\circ}$ case needs to have a height to width ratio of approximately $14,000 / 3,000 \approx 4.67$ whereas from Figure $7 \mathrm{~b}$ (for the $\phi=$ $180^{\circ}$ case) the PSD has a width to height ratio of approximately $12,000 / 3,000 \approx 4$. Although both cases require PSDs of similar size, the PSDs must be orientated in different directions. For the $\phi=90^{\circ}$ case, the PSD needs to be orientated with the long axis vertically whereas the $\phi=180^{\circ}$ system requires the long axis of the PSD to be horizontal.

Comparing the data shown in Figures 5 and $7 \mathrm{~b}$, it can be seen that the orientation of the trace of the laser on the PSD is different in both figures. In Figure 5 the traces on the PSD are convex while the traces in Figure $7 \mathrm{~b}$ are concave. The concavity of these curves is dependent on the angle of inclination of the PSD. If the PSD is inclined at an angle greater than $45^{\circ}$, as in Figure 5, the reflected laser maps out convex curves; if the PSD is inclined at an angle less than $45^{\circ}$, the traces are concave as shown in Figure $7 \mathrm{~b}$.

In both cases, once the system is properly characterized by the variables $\theta, \phi, D_{x}, D_{y}$, and $L$, each point on the PSD corresponds to a unique cantilever deflection and twist. As 
show in Figure 7, plots of the PSD/reflected laser intersection point show that as cantilevers are deflected from -10 to $+10 \mu \mathrm{m}$ and twisted from $-10^{\circ}$ to $10^{\circ}$, there are no overlapping curves. The only time these curves overlap is if there is a change in height in the $z$-direction (see Fig. 1) of the cantilever chip with respect to the incident laser. Some AFM systems such as the Veeco Multimode SPM (formally Digital Instruments) are arranged such that the sample is moved under the AFM cantilever allowing the optical beam deflection system to remain stationary with respect to the probe. In such a system it should be possible to determine the exact state (deflection and twist) of the cantilever simply from the PSD signal. Other systems such as the PicoSPM from Agilent Technologies (formally Molecular Imaging) have a geometry such that the probe is swept over a stationary sample. In such a case the optical beam deflection system becomes more complicated to interpret, and the state of the cantilever cannot be determined from a single PSD measurement.

\section{Discussion}

We have developed a model to characterize the simultaneous twist and deflection (originating from a point load force) of microcantilevers such as those used for performing lateral or FFM. The model was validated with a macrosized cantilever that was deflected and twisted by the use of an indexing head designed and made in-house. Analysis of the $\phi=90^{\circ}$ and $\phi=180^{\circ}$ systems shows that the $\phi=90^{\circ}$ geometry is equivalent to the standard $\phi=180^{\circ}$ system in accuracy and sensitivity.

In typical AFMs the optical beam deflection system is designed to facilitate the alignment of the optical beam on the cantilever. However, in these systems there is no way to infer the state of the cantilever based on the PSD signal. Although there are ways to calibrate the system to obtain the lateral deflection and twist of the cantilever based on the PSD signal, these methods can be complicated and difficult to implement. If AFMs were designed such that the variables of the system shown in Figure 1 were well defined, there would be no need to characterize the PSD signal. In fact, each PSD measurement would indicate the exact state of the cantilever that, provided the lateral and torsional spring constants were well defined, would give the torque and the normal force applied to the cantilever. This would allow users to obtain more accurate quantitative data with accuracy limited by the lateral and torsional spring constants.

\section{REFERENCES}

Beaulieu, L.Y., Godin, M., Laroche, O., Tabard-Cossa, V. \& Grütter, P. (2006). Calibrating laser beam deflection systems for use in atomic force microscopes and cantilever sensors. Appl Phys Lett 88, 083108.

Beaulieu, L.Y., Godin, M., Laroche, O., Tabard-Cossa, V. \& GrütTER, P. (2007). A complete analysis of the laser beam deflection systems used in cantilever-based systems. Ultramicroscopy 107, 422-430.

Binnig, G., Quate, C.F. \& Gerber, C. (1986). Atomic force microscope. Phys Rev Lett 56, 930-933.

Godin, M., Laroche, O., Tabard-Cossa, V., Beaulieu, L.Y., GrütTER, P. \& Williams, P.J. (2003). Combined in situ micromechanical cantilever-based sensing and ellipsometry. Rev Sci Instrum 74, 4902-4907.

Goto, M., Kasahara, A., Konishi, Y., Oishi, T., Tosa, M. \& Yoshihara, K. (2003). Frictional property of zinc oxide coating films observed by lateral force microscopy. Jpn J Appl Phys 42, 4834-4836.

Lantz, M.A., O'Shea, S.J., Hoole, A.C.F. \& Welland, M.E. (1997). Lateral stiffness of the tip and tip-sample contact in frictional force microscopy. Appl Phys Lett 70, 970-972.

Mate, C.M. (1995). Force microscopy studies of the molecular origins of friction and lubrication. IBM J Res Develop 39, 617-627.

Mate, C.M., McClelland, G.M., Erlandsson, R. \& Chiang, S. (1987). Atomic-scale friction of a tungsten tip on a graphite surface. Phys Rev Lett 59, 1942-1945.

Meyer, G. \& Amer, N.M. (1988). Novel optical approach to atomic force microscopy. Appl Phys Lett 53, 1045-1047.

Meyer, G. \& Amer, N.M. (1990). Simultaneous measurement of lateral and normal forces with an optical-beam-deflection atomic force microscope. Appl Phys Lett 57, 2089-2091.

Ogletree, F., Carpick, R.W. \& Salmeron, M. (1996). Calibration of frictional forces in atomic force microscopy. Rev Sci Instrum 67, 3298-3306.

Reissner, E. \& STEIn, M. (1951). Torsion and transverse bending of cantilever plates. Langley Research Center, NACA Technical Note 2369.

Ruan, J.A. \& Bhushan, B. (1994). Atomic-scale friction measurements using friction force microscopy: Part I-General principles and new measurement techniques. J Tribol Trans ASME 116, 378-388.

SARID, D. (1994). Scanning Force Microscopy: With Applications to Electric, Magnetic, and Atomic Forces. New York: Oxford University Press USA.

Tocha, E., Schönherr, H. \& VAncso, G.J. (2006). Quantitative nanotribology by AFM: A novel universal calibration platform. Langmuir 22, 2340-2350.

VArenberg, M., Etsion, I. \& Halperin, G. (2003). An improved wedge calibration method for lateral force in atomic force microscopy. Rev Sci Instrum 74, 3362-3367.

Yacoot, A., Koenders, L. \& Wolff, H. (2007). An atomic force microscope for the study of tip-sample interactions. Meas Sci Technol 18, 350-359. 